\title{
On the Estimate Measurement Uncertainty of the Insertion Loss in a Reverberation Chamber Including Frequency Stirring
}

\author{
Angelo Gifuni, Member, IEEE, Luca Bastianelli, Maurizio Migliaccio, Fellow, IEEE, Franco Moglie, Senior Member, IEEE, \\ Valter Mariani Primiani, Senior Member, IEEE, and Gabriele Gradoni, Member, IEEE
}

\begin{abstract}
In this paper, we present an improved model of measurement standard uncertainty (MU) of the insertion loss (IL) in a reverberation chamber (RC), including frequency stirring (FS). Differently from the previous model, the enhanced one does not require specific conditions on the parameter to be measured. Such an enhancement is applicable for all usable measurement conditions in RCs. Moreover, a majorant is derived, that is obtained under a weak condition on the coefficient of variation (CV) of the parameter to be measured. Results from measurements support the validity of both the proposed enhancement and the majorant.
\end{abstract}

Index Terms - Reverberation chamber (RC), frequency stirring (FS), mechanical stirring (MS), measurement uncertainty (MU).

\section{INTRODUCTION}

Measurement uncertainty (MU) quantification is important to improve the applications of reverberation chambers (RCs) [1]. Hybrid stirring increases the number of uncorrelated samples and, consequently, reduces the MU [1]-[7]. In this paper, we consider the hybrid stirring as realized by a combination of frequency stirring (FS) and mechanical stirring (MS) [2]-[3]. The FS measurements also allow us to represent the measured quantity in time domain [8]-[10]. The MU of the insertion loss (IL) in an RC with hybrid MS and FS was addressed in [11], where a model was developed and achieved under conditions of well-stirred fields. We refer to this model as the previous model. In [11], MU is estimated following the approach described in [12], hence considering it as a type A uncertainty. This type of uncertainty is normally the main component for $\mathrm{MU}$ in RCs [13]. The type B evaluation uncertainty depends on Manufacturer's specifications of the instrumentations, as well as on the

Manuscript received May 12, 2018; revised Xxxx, xx, 2018.

A. Gifuni and M. Migliaccio are with the Dipartimento di Ingegneria, Università di Napoli Parthenope, Centro Direzionale di Napoli, Napoli 80143, Italy (e-mail: angelo.gifuni@uniparthenope.it; maurizio.migliaccio@uniparthenope.it).

L. Bastianelli, F. Moglie, and V.M. Primiani are with the Dipartimento di Ingegneria dell'Informazione, Università Politecnica delle Marche, 60131 Ancona, Italy (e-mail: f.moglie@univpm.it; 1.bastianelli@pm.univpm.it; v.mariani@univpm.it).

G. Gradoni is with the School of Mathematical Sciences and with the George Green Institute for Electromagnetics Research, Department of Electrical and Electronics Engineering, University of Nottingham, Nottingham NG7 2RD, U.K. (e-mail: gabriele.gradoni@nottingham. ac.uk). specific calibration procedure used for measurements, which can change from case to case. However, instrumentation and setting adopted in our measurements are also shown here. An extensive treatment of the MU in RCs was addressed in [13], where an approach similar to that in [11] was used, as it will be specified below. Nevertheless, some meaningful differences in the approaches should be discussed. We will discuss this in section $\mathrm{V}$. The purpose of this paper is to enhance the previous model and its range of usability. The enhanced model does not require specific conditions for its validity; it is de facto a generalization of the previous model. It is found that such an enhancement is applicable for all usable measurement conditions of IL in RCs including conditions at low frequencies. A majorant of the standard MU is also obtained: it requires the coefficient of variation (CV) of the measured samples to be less than or equal to one. We find that the majorant retrieves the previous model. It can be applied when a conservative margin for statistical fluctuations is considered and the abovementioned $\mathrm{CV}$ is less than one.

\section{THEORY}

We develop the enhanced theory by considering the IL as in [11]. We can write [11]:

$$
I L=\left\langle\left|S_{21}\right|^{2}\right\rangle_{N}=\left\langle E^{2}\right\rangle_{N},
$$

where $<>_{N}$ represents the ensemble average with respect to the $N$ uncorrelated field configurations from MS, e.g., performed through metallic stirrer(s) only. $E^{2}$ represents the squared amplitude of the transmission coefficient $S_{21}$; it is a random variable $(\mathrm{RV})$. Actually, $I L$ is a sample mean (SM) and therefore has statistical fluctuations: it is an RV. We can write the mean, variance, and $\mathrm{CV}$ of the RV $I L_{f}$, respectively, as follows ${ }^{1}[11]$ :

$$
\begin{aligned}
& \mu_{I L_{f}}=I L_{f, 0}, \\
& \sigma_{I L_{f}}^{2}=\frac{\sigma_{E^{2}, f, 0}^{2}}{N}, \\
& \delta_{I L_{f}}^{2}=\frac{\sigma_{I L_{f}}^{2}}{I L_{f, 0}^{2}},
\end{aligned}
$$

\footnotetext{
${ }^{1}$ Differently from [11], here, the mean squared of the IL, as well as other squared means, is written with no brackets.
} 
where $N$ is the number of uncorrelated samples used to estimate the SM $I L_{f}, f$ is the frequency, and $\sigma_{E^{2}, f, 0}^{2}$ is the variance of $E^{2}$. Note that the parameters of the RVs are marked by the subscript 0 as in [11]. In order to analyze the behavior of the enhanced model, we will use the following conditions:

$$
\begin{aligned}
& \delta_{E^{2}, f, 0}=\frac{\sigma_{E^{2}, f, 0}}{\mu_{I L_{f}}}=\frac{\sigma_{E^{2}, f, 0}}{I L_{f, 0}}=\text { constant in FSB }, \\
& \delta_{E^{2}, f, 0}=\frac{\sigma_{E^{2}, f, 0}}{\mu_{I L_{f}}}=\frac{\sigma_{E^{2}, f, 0}}{I L_{f, 0}} \leq 1, \\
& \delta_{E^{2}, f, 0}=\frac{\sigma_{E^{2}, f, 0}}{\mu_{I L_{f}}}=\frac{\sigma_{E^{2}, f, 0}}{I L_{f, 0}}>1,
\end{aligned}
$$

where FSB means frequency stirring bandwidth and $\delta_{E^{2}, f, 0}$ is the $\mathrm{CV}$ of $E^{2}$. Note that for well-stirred fields we have $\mu_{I L_{f}}=I L_{f, 0}=\sigma_{E^{2}, f, 0}$. In general, it turns out that $\mu_{I L_{f}}=I L_{f, 0} \geq \sigma_{E^{2}, f, 0}$, i. e., the condition (5b) is met in most cases for fields in RCs; often, both the conditions (5a) and (5b) are met. However, at low frequencies, both the exponential and the Rician distribution could be unmet, as well as both conditions (5a) and (5b). We want to develop a model valid under any condition (5a)-(5c). When the condition (5b) is met, the IL includes a direct (line-of-sight, or LOS) component, which can be both desirable [14] and undesirable: the latter is the typical case of RCs loaded [15][16]. When the samples are acquired both by mechanical and frequency stirring ${ }^{2}$, then $I L_{f}$ is denoted by $I L_{N, \Delta f}$ and we can write [11]:

$$
I L_{N, \Delta f}=W=\left\langle\left\langle E^{2}\right\rangle_{N}\right\rangle_{k}=I L_{\Delta f, N}=\left\langle\left\langle E^{2}\right\rangle_{k}\right\rangle_{N},
$$

where the subscript $\Delta f$ indicates that the averages are made over $k$ uncorrelated frequency samples in FSB [11]. Here, we consider the averages with respect to $N$ first and then those with respect to $k$ [11]; it is implicit that we consider steptuned RCs [17]-[18]. The averages for each frequency point correspond to SMs including only the MS from to metallic stirrer(s). Such SMs are assumed to be uncorrelated RVs and they are denoted by $I L_{f 1}, I L_{f 2}, \cdots, I L_{f k}$. Their corresponding mean values are denoted by $I L_{f 1,0}, I L_{f 2,0}, \cdots, I L_{f k, 0}$. The RV $W$ given by (6) can be expressed as follows [11]:

$$
W=\frac{1}{k}\left[I L_{f 1}+I L_{f 2}+\cdots+I L_{f k}\right] .
$$

Note that $\Delta f=f_{k}-f_{1}$, where $f_{1}$ and $f_{k}$ are the minimum and the maximum frequency of the FS. We are interested in the mean and variance of $W$. We can write:

$$
\begin{aligned}
& \mu_{W}=W_{0}=\frac{1}{k}\left[I L_{f 1,0}+I L_{f 2,0}+\cdots+I L_{f k, 0}\right], \\
& \sigma_{W}^{2}=\frac{1}{k N}\left\{\frac{\sigma_{E^{2}, f 1,0}^{2}+\sigma_{E^{2}, f 2,0}^{2}+\cdots+\sigma_{E^{2}, f k, 0}^{2}}{k}\right\} .
\end{aligned}
$$

\footnotetext{
${ }^{2}$ The MS considered in (6) is limited to metallic stirrer(s).
}

We want to transform (9a) so that it gives a significant connection between MS and FS. We can write:

$$
\sigma_{W}^{2}=\frac{1}{k N}\left\{\begin{array}{l}
\frac{\delta_{E^{2}, f 1,0}^{2} I L_{f 1,0}^{2}+\delta_{E^{2}, f 2,0}^{2} I L_{f 2,0}^{2}}{k} \\
\frac{+\cdots+\delta_{E^{2}, f k, 0}^{2} I L_{f 2,0}^{2}}{k}
\end{array}\right\},
$$

where $\delta_{E^{2}, f, 0}^{2}=\sigma_{E^{2}, f, 0}^{2} / I L_{f, 0}^{2}$. The mean $I L_{f i, 0}^{2}(i=1,2, \ldots, k)$ changes as the frequency changes; the variations depend on the RC and FSB. The means $\delta_{E^{2}, f, 0}^{2}$ and $I L_{f, 0}^{2}$, as well as the corresponding sample estimates, can be thought of as two $\mathrm{RVs}$, whose values are associated by the frequencies $f i$. We can write [19], [20]:

$$
\left\langle\delta_{E^{2}, f, 0}^{2} I L_{f, 0}^{2}\right\rangle_{k}=\delta_{E^{2}, \Delta f, 0}^{2} I L_{\Delta f, 0}^{2}+\left[\operatorname{Cov}\left(\delta_{E^{2}, f, 0}^{2}, I L_{f, 0}^{2}\right)\right]_{k},
$$

where $\delta_{E^{2}, \Delta f, 0}^{2}$ and $I L_{\Delta f, 0}^{2}$ are the means of $\delta_{E^{2}, f, 0}^{2}$ and $I L_{f, 0}^{2}$ in the FSB, respectively; Cov means covariance; the subscript $k$ means that the concerning parameter is referred to the FSB. The covariance is equal to zero when the $\mathrm{RVs}$ are uncorrelated or when (5a) is met; in the latter case, we can write: $\delta_{E^{2}, 0}^{2}=$ const. $=\delta_{E^{2}, \Delta f, 0}^{2}$. The RVs $\delta_{E^{2}, f, 0}^{2}$ and $I L_{f, 0}^{2}$, which are estimated by corresponding sample means from $N$ uncorrelated sampling data of $S_{21}$, are not totally uncorrelated in practice as the former includes an effect of the latter. However, when they are sufficiently uncorrelated (10a) can be well approximated as follows:

$$
\left\langle\delta_{E^{2}, f, 0}^{2} I L_{f, 0}^{2}\right\rangle_{\mathrm{FSB}}=\delta_{E^{2}, \Delta f, 0}^{2} I L_{\Delta f, 0}^{2} .
$$

It is highlighted that (10b) is valid also in case of sample estimates. We can write [11]:

$$
W_{0}^{2}+\sigma_{\Delta f, 0}^{2}=\left[\frac{I L_{f 1,0}^{2}+I L_{f 2,0}^{2}+\cdots+I L_{f k, 0}^{2}}{k}\right],
$$

where $\sigma_{\Delta f, 0}$ is the standard deviation of the means $I L_{f 1,0}, I L_{f 2,0}$, $\cdots, I L_{f k, 0}$.

\section{A. Enhanced Model: Valid Under any Condition (5a)-(5c)}

Manipulating (9), (10b), and (11), we can write:

$$
\begin{aligned}
& \sigma_{W}=\frac{W_{0}}{\sqrt{k N}}\left(\delta_{E^{2}, \Delta f, 0}^{2}\right)^{1 / 2} \sqrt{1+\delta_{\Delta f, 0}^{2}}, \\
& \delta_{W}=\left(\delta_{E^{2}, \Delta f, 0}^{2}\right)^{1 / 2} \frac{\sqrt{1+\delta_{\Delta f, 0}^{2}}}{\sqrt{k N}},
\end{aligned}
$$

where $\sigma_{W}$ and $\delta_{W}$ are the standard $\mathrm{MU}$ and the relative standard MU of $W$, respectively; the CV $\delta_{\Delta f, 0}=\sigma_{\Delta f, 0} / W_{0}$. When (5a) is met and $\delta_{E^{2}, 0}=1$, which corresponds to the case of well-stirred fields, (12) and (13) become equal to (10) and (13) in [11], respectively, as expected. Therefore, the term $\left(\delta_{E^{2}, \Delta f, 0}^{2}\right)^{1 / 2}$ defines an enhancement of the previous model. Practically, $\sigma_{W}$ and $\delta_{W}$ are also RVs as parameters on the right side of (12) and (13), as well as those in the 
equations below, are sample estimates. They depend however on $N$, in which case we omit the zero at their subscript.

\section{B. Variation of the Enhanced Model: Valid Under Condition} (5a)

When (5a) is met, a variation of the enhanced model (12)(13) can be obtained; in fact, (12) and (13) became as follows [21]:

$$
\begin{aligned}
& \sigma_{W}=\frac{W_{0}}{\sqrt{k N}} \delta_{E^{2}, \Delta f, 0} \sqrt{1+\delta_{\Delta f, 0}^{2}}, \\
& \delta_{W}=\delta_{E^{2}, \Delta f, 0} \frac{\sqrt{1+\delta_{\Delta f, 0}^{2}}}{\sqrt{k N}},
\end{aligned}
$$

where $\delta_{E^{2}, \Delta f, 0}=\delta_{E^{2}, f, 0}$ for the assumption (5a). Since population parameters are estimated by the corresponding sample statistics ${ }^{3}$, which uses $N$ uncorrelated sampling data of $S_{21}$, we can de facto know if (5a) is met only when $N$ is much greater than one: in such cases, the statistical fluctuations are substantially reduced. When $N$ is not much greater than one, we can assume that (5a) is met and calculate its average in the FSB; the comparison of the results with those from measurements proves if the assumption holds true.

\section{Some Detail on the Behavior of the Enhanced Model and its Variation}

Note that $\delta_{E^{2}, \Delta f, 0}$ and $\left(\delta_{E^{2}, \Delta f, 0}^{2}\right)^{1 / 2}$ are mean and root mean square (RMS) of $\delta_{E^{2}, f, 0}$ in the FSB. They tend to the same value when the variance of $\delta_{E^{2}, f, 0}$ or of the concerning sample estimate tends to zero in the FSB. In section IV, it is shown that when $N$ is greater than or equal to eight, (12)-(13) practically give the same results of (14)-(15). When $N$ is less than eight, results from (14)-(15) give smaller values than the corresponding ones from (12)-(13), as expected. It will be shown that results from (12)-(13) match those from measurements. Moreover, on equal $N$ value, the difference between results from (12)-(13) and those from (14)-(15) is maximum when the $K$-Factor is zero. By measurements, which are the samples of $S_{21}$ taken in the RC at frequencies $f_{i}$ $(i=1,2, \ldots, k)$ within the FSB, one can estimate the means $I L_{f 1,0}, I L_{f 2,0}, \cdots, I L_{f k, 0}$, as well as $W_{0}^{2}, \delta_{E^{2}, \Delta f, 0}$, and $\delta_{\Delta f, 0}^{2}$. The variances are estimated as sample variances. Then, by using (12)-(15), we can calculate the corresponding standard and relative standard MUs. For $k=1$, the achieved models retrieve the pure MS model of (3) and (4), of which they are extensions. Since the means $I L_{f 1,0}, I L_{f 2,0}, \cdots, I L_{f k, 0}$ are estimated by the corresponding sample means, their statistical fluctuations increase with the decrease of $N$; in particular, both $\delta_{E^{2}, \Delta f, 0}$ and $\left(\delta_{E^{2}, \Delta f, 0}^{2}\right)^{1 / 2}$ are appreciably underestimated

\footnotetext{
${ }^{3}$ This is the reason why the symbol $\delta_{E^{2}, \Delta f, 0}$ is used in (14) and (15) instead of $\delta_{E^{2}, f, 0}$.
}

when $N$ is small [13]. It will be confirmed by results in section IV.

\section{D.Models Including the Effect of Statistical Non-Uniformity}

By following assumptions and developments in [11, after eq. 14], we can write:

$$
\sigma_{W_{m p}}^{2}=\delta_{E^{2}, \Delta f, 0}^{2} \frac{W_{m p, 0}^{2}}{p k N}\left(1+\delta_{s p, p, 0}^{2}\right)\left(1+\delta_{\Delta f, 0}^{2}\right)+\frac{\sigma_{s p, p, 0}^{2}}{p},
$$

where the subscripts $p, m p$, and $s p$ mean $p$ independent positions of at least one of the two antennas, multiple positions, and a single position, respectively; $\sigma_{s p, p, 0}^{2}$ is the variance due to the lack of perfect uniformity [11]. Note that the constancy of $\delta_{E^{2}, A f, 0}$ for all positions $p$ is acceptable in this context. If $k=1$ (only MS), then (16) becomes as follows:

$$
\sigma_{W_{m p}}^{2}=\delta_{E^{2}, f, 0}^{2} \frac{W_{m p, 0}^{2}}{p N}\left(1+\delta_{s p, p, 0}^{2}\right)+\frac{\sigma_{s p, p, 0}^{2}}{p} .
$$

It is useful to recast (16) as:

$$
\sigma_{W_{m p}}^{2}=\delta_{E^{2}, \Delta f, 0}^{2} \frac{W_{m p, 0}^{2}}{p k N} C F_{0}+\frac{\sigma_{s p, p, 0}^{2}}{p},
$$

where

$$
C F_{0}=\left(1+\delta_{\Delta f, 0}^{2}\right)\left(1+\delta_{s p, p, 0}^{2}\right) .
$$

It is also useful to introduce the ratio $R_{\delta s q, 0}=\delta_{s p, p, 0}^{2} / \delta_{\Delta f, 0}^{2}$. If $R_{\delta s q, 0}<<1$, then $C F_{0} \cong\left(1+\delta_{\Delta f, 0}^{2}\right)$. We can write:

$$
\sigma_{W_{m p}}=\sqrt{\delta_{E^{2}, \Delta f, 0}^{2} \frac{W_{m p, 0}^{2}}{p k N} C F_{0}+\frac{\sigma_{s p, p, 0}^{2}}{p}}=\sqrt{\sigma_{1}^{2}+\sigma_{2}^{2}},
$$

with

$$
\begin{aligned}
& \sigma_{1}=\delta_{E^{2}, \Delta f, 0} \frac{W_{m p, 0}}{\sqrt{p k N}} \sqrt{C F_{0}}, \\
& \sigma_{2}=\sigma_{s p, p, 0} / \sqrt{p} .
\end{aligned}
$$

Equations (21) and (22) allow to estimate standard MU contributions $\sigma_{1}$ and $\sigma_{2}$. However, the two quantities are not completely uncorrelated [11]. It is important to note that the value of $\delta_{E^{2}, \Delta f, 0}$ mostly affects $\sigma_{1}$; that is, it affects (12)-(15). The total relative MU can be written as follows:

$$
\begin{aligned}
\delta_{W_{m p}} & =\sqrt{\delta_{E^{2}, \Delta f, 0}^{2} \frac{C F_{0}}{p k N}+\frac{\sigma_{s p, p, 0}^{2}}{W_{m p, 0}^{2} p}} \\
& =\sqrt{\delta_{E^{2}, \Delta f, 0}^{2} \frac{C F_{0}}{p k N}+\frac{\delta_{s p, p, 0}^{2}}{p}}=\sqrt{\sigma_{1, r}^{2}+\sigma_{2, r}^{2}},
\end{aligned}
$$

where $\sigma_{1, r}^{2}$ and $\sigma_{2, r}^{2}$ are the contributions to the relative $\mathrm{MU}$, which correspond to the uncertainties squared $\sigma_{1}^{2}$ and $\sigma_{2}^{2}$, respectively.

\section{E. Majorant of the Enhanced Model: Valid Under Condition} (5b)

Note that if $(5 b)$ is met, we can write: 


$$
\begin{aligned}
\sigma_{W_{c}} & \leq \frac{W_{c, 0}}{\sqrt{k N}} \sqrt{1+\delta_{c, \Delta f, 0}^{2}}, \\
\delta_{W_{c}} & \leq \frac{\sqrt{1+\delta_{c, \Delta f, 0}^{2}}}{\sqrt{k N}} .
\end{aligned}
$$

Interestingly, the right sides of (24) and (25) are the same as in the previous model, and they give majorants of the corresponding standard MUs. It is specified that the subscript $c$ in (24) and (25) denote that fields meet the condition (5b). It is important to emphasize that (12) and (13), as well as the corresponding (20) and (23), are a general model for the standard MU of the IL in RCs, i.e., (10)-(13), as well as (24) and (27) in [11], are a particular case of (12)-(13) and (20) and (23), respectively, which are retrieved when $\delta_{E^{2}, 0}=1$.

\section{F. Connection between the $C V$ of $E^{2}$ and the K-Factor}

Finally, it is useful to express the $\mathrm{CV} \delta_{E^{2}, f, 0}$ by the $K$-Factor,

which is denoted by $K_{f, 0}$. When the transmission coefficient $S_{21}$ has a Rician distribution, we can write:

$$
\delta_{E^{2}, f, 0}=\sqrt{\frac{2 K_{f, 0}+1}{2 K_{f, 0}+1+K_{f, 0}^{2}}},
$$

where

$$
K_{f, 0}=\left(\mu_{1, f, 0}^{2}+\mu_{2, f, 0}^{2}\right) / 2 \sigma_{E^{2}, f, 0}^{2},
$$

$\mu_{1, f, 0}$ and $\mu_{2, f, 0}$ are the means of the real and imaginary part, respectively, of the coefficient $S_{21}$, and $\sigma_{E}^{2} f, 0$ is the variance of those parts. From (26), one notes that if $K_{f, 0}$ is constant in the FSB then also $\delta_{E^{2}, f, 0}$ is constant.

\section{MEASUREMENTS SETUP}

Measurements are made in the RC at Università Politecnica delle Marche, Ancona, Italy, which is operated in step mode to produce the measurements used in this paper. The measurement setup and acquisition settings are the same as in [11], except that in this case two type of configurations of the antennas are used for measurements: one configuration minimizes the direct coupling between the antennas, which are distant and cross-polarized, and the other one maximizes it. In the latter case, the antennas are on the LOS at a known distance from each other; they are tip-to-tip positioned and co-polarized; several distances are used for measurements but only results concerning the distances of $0.05 \mathrm{~m}$ and $0.3 \mathrm{~m}$ are shown for the sake of brevity. The former and latter measurement configurations are here called A and B, respectively. It is specified that the measurement setup includes a four-port VNA, model Agilent 5071B, and two antennas, model Schwarzbeck Mess-Elektronik USLP 9143, whose usable frequency range (FR) ranges from $250 \mathrm{MHz}$ to $7 \mathrm{GHz}$ for EMC tests. The IF bandwidth and source power, which determine the instrument measurement uncertainty along with the set FR and amplitude of the measured transmission coefficient, are set to $3 \mathrm{kHz}$ and $0 \mathrm{dBm}$, respectively. It is specified that the standing wave ratio (SWR) of the antennas in free space is less than or equal to 1.75 from $250 \mathrm{MHz}$ to $8.2 \mathrm{GHz}$ and it is 3 , which is the maximum value, at $200 \mathrm{MHz}$. Note that corrections for impedance mismatches, which are important in the RC at low frequencies, are not necessary for the verification of the proposed model [11]. Hence, measurements are extended to $200 \mathrm{MHz}$ to test the model where the CV of $E^{2}$ can fluctuate around the unity. Over the FR from $0.2 \mathrm{GHz}$ to $8.2 \mathrm{GHz}$, 16,001 frequency points are acquired with a step frequency (SF) of $500 \mathrm{kHz}$ for a number of mechanical positions $M=64$ [11]. Note that the number 64 corresponds to the total number of acquired stirrer positions, which in turns corresponds to the total number of acquired (frequency) sweeps $(M=64)$ [11]. The total sweeps are divided in $n$ sets of (frequency) sweeps, so that each set includes $N$ sweeps and $M=n \cdot N$. The settings $n$ and $N$ can be changed to test the enhanced model [11]. For each sweep, the total number of processed frequency points $\kappa=16,000$ is divided in $q$ sets of frequencies, so that $\Delta f=(k-1) \cdot \mathrm{SF}$ and $\kappa=k \cdot q$. Differently from the notation in [11], the symbol for the total frequency points is denoted here by $\kappa$ to avoid confusion with the symbol of the $K$-Factor. The value of $q$ is the number of FSB or $\Delta f$ included in the FR. The mean $W_{0}$ in (12) is estimated $n$ times and the standard deviation of such $n$ averages $W_{i}(i=1$, $2, \cdots, n)$ is calculated [11]. The calculated standard deviation is an estimate of the measured standard uncertainty. When such an uncertainty is normalized to the average of the averages $W_{i}$, an estimate of the relative standard uncertainty is obtained. The measured standard MU is compared to the corresponding expected standard MUs, which are obtained by applying (12), as well as (14), and (24). They are applied by using any of the $n$ estimates $W_{i}$ and the corresponding estimates of $\sigma_{\Delta f, 0}^{2},\left(\delta_{E^{2}, \Delta f, 0}^{2}\right)^{1 / 2}$, and $\delta_{E^{2}, \Delta f, 0}$; clearly, the estimates of $\left(\delta_{E^{2}, \Delta f, 0}^{2}\right)^{1 / 2}$ and $\delta_{E^{2}, \Delta f, 0}$ are also calculated $n$ times, and they are used in (12) and (14), respectively, as mentioned above. Similarly, the measured relative standard MU is compared to the corresponding expected relative standard MUs, which are obtained by applying (13), (15), and (25). The unorrelation of samples is verified by calculating the autocorrelation function (ACF). Here, the uncorrelation threshold used is $1 / e$, where $e$ is the Neper's number. Thresholds of 0.5 and 0.7 could be also used [22]. However, the higher the threshold the higher the residual correlation of samples [21]. Note that the 64 frequency sweeps of each IL measurement can be thought as a matrix of 64 rows and 16,001 columns, where only the frequency changes (FS) along each row, whereas only the stirrer position (MS) changes along each column. The ACF is calculated for both row and column. For measurements where the IL includes a significant variable LOS component, the ACF is considerably affected. A short sequence of frequency samples, where the average of the direct component is removed, could be considered, as made in [21]; this method has the drawback to use only a few samples for the estimate of the ACF and, however, it is unreliable [21]. Here, the direct component is removed before we calculate the ACF for measurements concerning the configuration $\mathrm{B}$; it is removed for each frequency point, i.e., it is removed for both MS and FS. The direct component to be removed is obtained by using all 64 
sweeps. For both measurements from configuration A, where it is not necessary to remove the residual direct component, and measurements from configuration $\mathrm{B}$, acceptable results are obtained according to the abovementioned threshold, which are not explicitly shown here for the sake of brevity. However, to ensure uncorrelated samples in all the FR and for any FSB, a decimation of samples from 1 through 8 is made for samples concerning the configuration B. Similarly, a decimation of samples from 1 through 2 is made for samples concerning the configuration A. Hence, SF becomes $1 \mathrm{MHz}$ for configuration A measurements and $4 \mathrm{MHz}$ for configuration B measurements. Finally, we note that when an appreciable direct component is not present or when it is removed and the stirred component dominates, the uncorrelation can be verified by using the correlation coefficient (CC) applied to the amplitude squared of samples [23]. By using such a method, it is confirmed that the results deteriorate when the FSB increases, as well as when it is too small, with respect to the number of samples [23].

\section{RESULTS}

The effect of the enhancement of the previous model and of the majorant is well-visible in $\sigma_{1}$. Therefore, in order to make the verification of the proposed models effective and simple, we use (12)-(13), (14)-(15), and (24)-(25). Fig. 1 and 2 show the standard MUs and the relative standard MUs given by (12), (14), (24), and (13), (15), (25), respectively, for measurements concerning the configuration A. Note that $\Delta f=$ $(k-1) \cdot 1 \mathrm{MHz}$. Fig. 3 shows a detail of Fig. 2 at low frequencies. From Fig. 4 to Fig. 7, the $\mathrm{CV} \delta_{\Delta f}$, the $\mathrm{CV} \delta_{E^{2}, f}$ along with its average value and RMS value, and $K$-Factor are shown; in particular, Fig. 6 shows a detail of Fig. 5 at low frequencies. All processing settings $(N, k$, etc.) are reported in the figure captions.

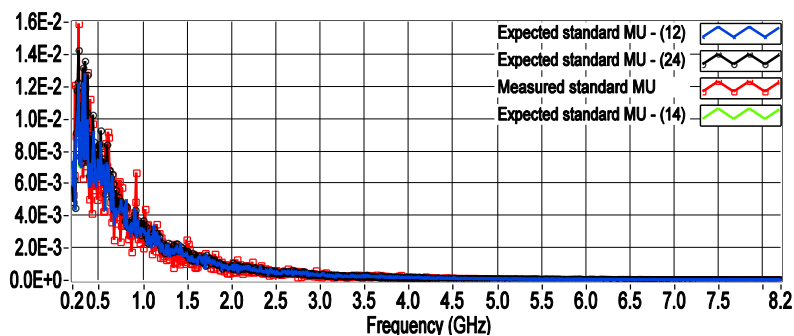

Fig. 1. Standard MU from the configuration A; for measured and expected standard uncertainties, $M=64, \kappa=8,000, N=8, n=8$, and $k=10(\Delta f=9$ $\mathrm{MHz})$.

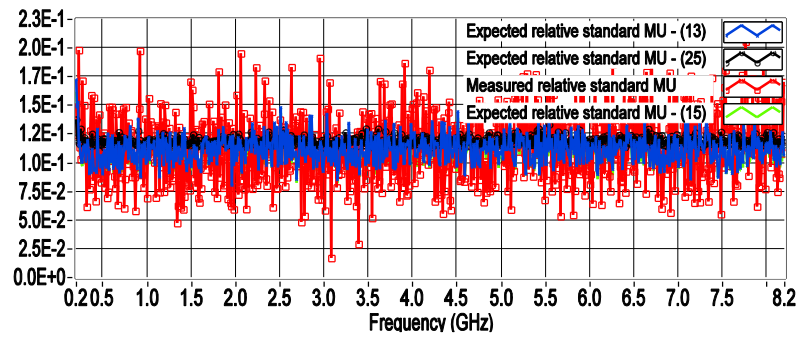

Fig. 2. Relative standard MU from the configuration A; for measured and expected relative standard uncertainties, $M=64, \kappa=8,000, N=8, n=8$, and $k=10(\Delta f=9 \mathrm{MHz})$
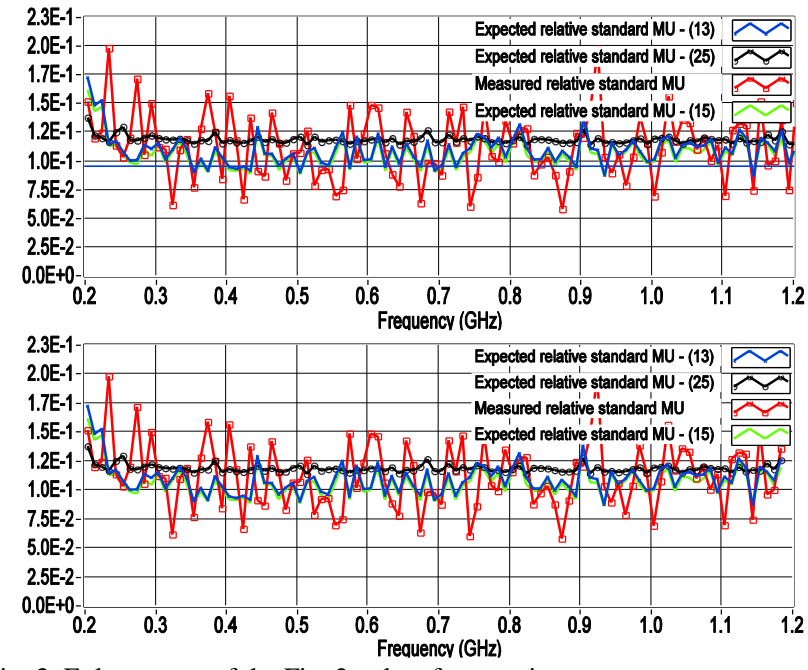

Fig. 3. Enlargement of the Fig. 2 at low frequencies.

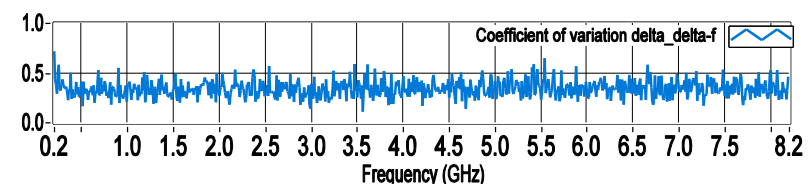

Fig. 4. $\mathrm{CV} \delta_{\Delta f}$ from the configuration $\mathrm{A} ; N=8, k=10(9 \mathrm{MHz})$.

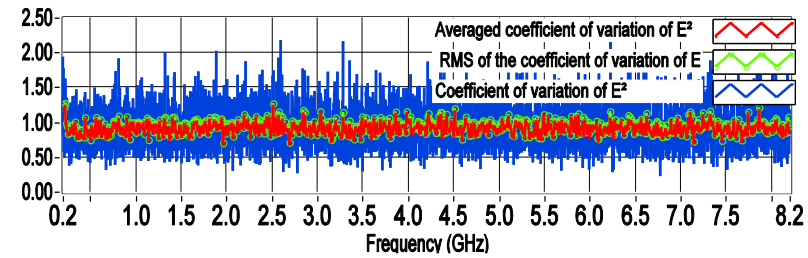

Fig. 5. CV $\delta_{E^{2}, f}$ from the configuration A, its average value, and RMS

value in the FSB; for all traces, $M=64, \kappa=8,000$, and $N=8 . k=1$ for blue and unmarked trace and $k=10(\Delta f=9 \mathrm{MHz})$ for the green and red traces, which are cross-marked and circled-marked, respectively.

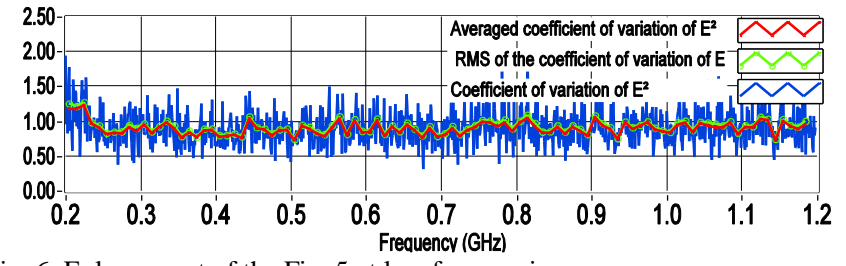

Fig. 6. Enlargement of the Fig. 5 at low frequencies.

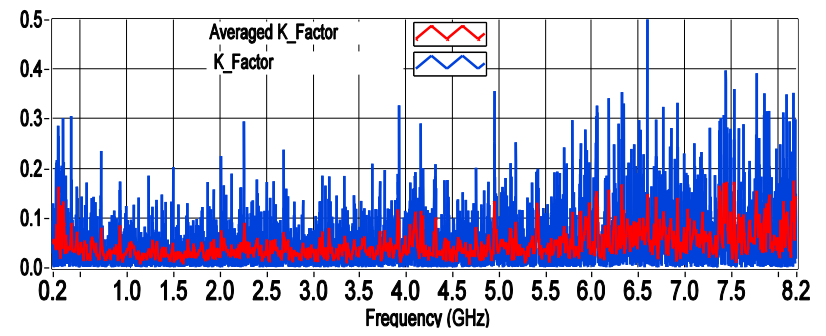

Fig. 7. $K$-Factor from the configuration A; for both traces $M=64, \kappa=$ 8,000 , and $N=64 . k=1$ for blue and unmarked trace and $k=10(\Delta f=9$ $\mathrm{MHz}$ ) for red and cross-marked trace. 


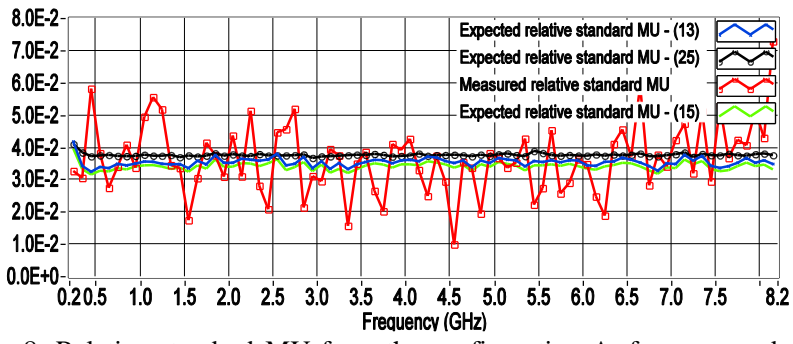

Fig. 8. Relative standard MU from the configuration A; for measured and expected relative standard uncertainties, $M=64, \kappa=8,000, N=8, n=8$, and $k=100(\Delta f=99 \mathrm{MHz})$.

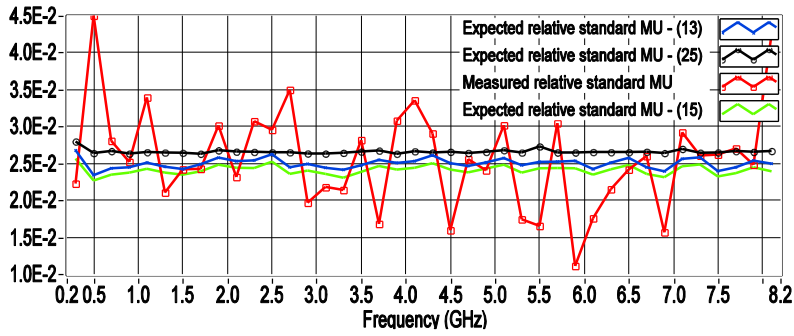

Fig. 9. Relative standard MU from the configuration A; for measured and expected relative standard uncertainties, $M=64, \kappa=8,000, N=8, n=8$, and $k=200(\Delta f=199 \mathrm{MHz})$.

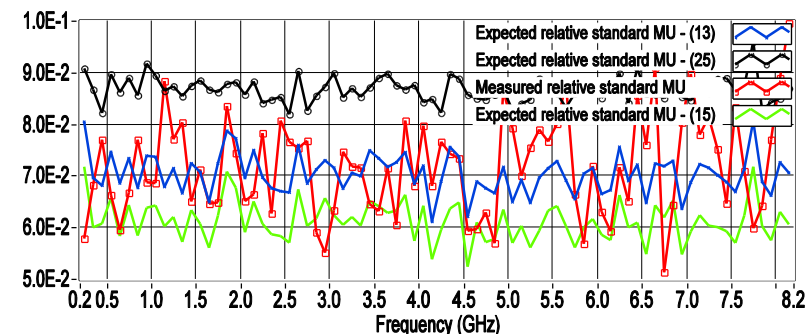

Fig. 10. Relative standard MU from the configuration A; for measured and expected relative standard uncertainties, $M=64, \kappa=8,000, N=2, n=32$, and $k=100(\Delta f=99 \mathrm{MHz})$.

All Figs show the expected statistical fluctuations. The comparison between measured standard MUs and corresponding expected standard MUs shows that (12)-(13), as well as (14)-(15) are supported by measurement results. In order to prove that the models works also for different FSBs, expected relative standard MSs are shown in Fig. 8 and Fig. 9 , where $k=100(\Delta f=99 \mathrm{MHz})$ and $k=200(\Delta f=199$ $\mathrm{MHz}$ ), respectively. It is also confirmed that expected results from (24) and (25) are the same as those from (12) and (13), respectively, when $K=0$, which implies $\delta_{E^{2}}=1$ (the equal sign has to be taken in (24)-(25)), except at the low frequencies $(f<250 \mathrm{MHz})$, where a deviation is expected and observed (see Fig. 3). By Figs. (1)-(9), it is also noted that (12)-(13) and (14)-(15) give practically the same results for $N$ $=8$. In Fig. 10, where $N$ is 2, it is well visible the difference between results from (13) and (15). Such a difference is due to the statistical fluctuations, which increase as $N$ decreases, as mentioned above; the same applies to (12) and (14). The slight difference between results from (13) and (25), which is visible in Fig. 10, as well as those between results from (12) and (24), when in (24)-(25) the equal sign has to be taken, is due to the $N$ value; it decreases as $N$ increases because both
$\delta_{E^{2}, \Delta f, 0}$ and $\left(\delta_{E^{2}, \Delta f, 0}^{2}\right)^{1 / 2}$ are underestimated when $N$ is small, as mentioned above. It is important to note that the measured standard MU and the expected standard MU from (12), as well as the corresponding relative standard MUs, match also when $N$ is small $(N<4)$ for the effect of such an underestimate (see Fig. 10 for the relative standard MUs); otherwise, the abovementioned difference is acceptable from $N=4$ [11]. Figures from 11 to 15 show results of measurements concerning the configuration $B$ for $d=0.05 \mathrm{~m}$. In particular, Figs. 11 and 12 show expected standard MUs and expected relative MUs along with the corresponding measured MUs. The FSB is $96 \mathrm{MHz}$. One notes that expected and measured results match again. Note that (24) and (25) are clearly majorants of the corresponding measured uncertainties in these cases.

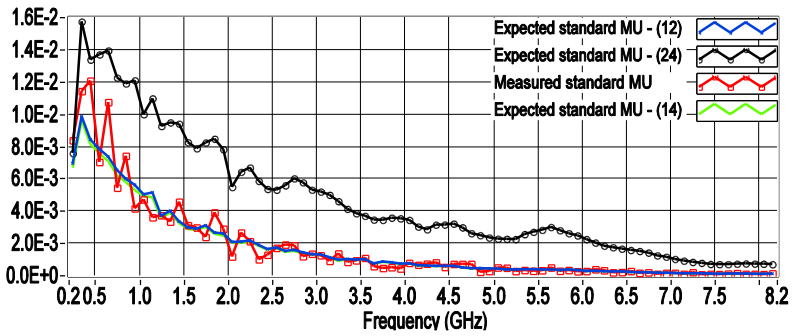

Fig. 11. Standard MU from the configuration $B, d=0.05 \mathrm{~m}$; for measured and expected standard uncertainties, $M=64, \kappa=2,000, N=8, n=8$, and $k$ $=25(\Delta f=96 \mathrm{MHz})$.

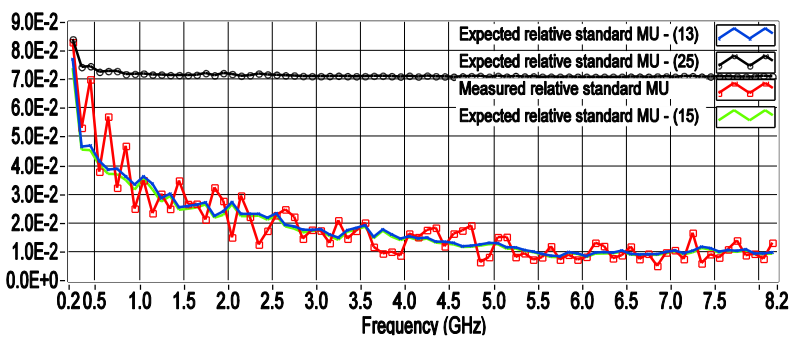

Fig. 12. Relative standard $\mathrm{MU}$ from the configuration $\mathrm{B}, \mathrm{d}=0.05 \mathrm{~m}$; for measured and expected standard uncertainties, $M=64, \kappa=2,000, N=8, n$ $=8$, and $k=25(\Delta f=96 \mathrm{MHz})$.

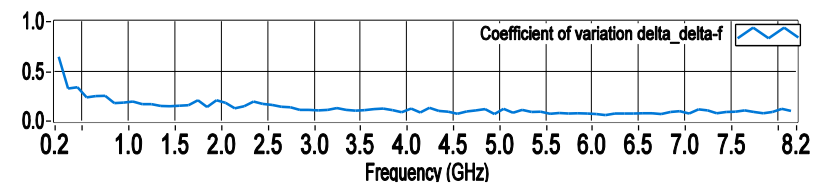

Fig. 13. $\mathrm{CV} \delta_{\Delta f}$ from the configuration $\mathrm{B}, \mathrm{d}=0.05 \mathrm{~m} ; N=8, k=25(96$ $\mathrm{MHz})$.

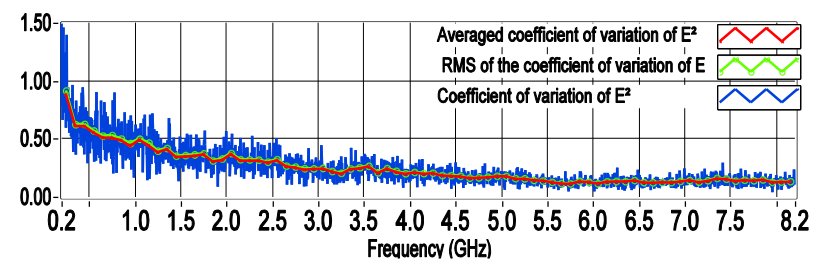

Fig. 14. $\mathrm{CV} \delta_{E^{2}, f}$ from the configuration $\mathrm{B}(\mathrm{d}=0.05 \mathrm{~m})$, its average value, and RMS value in the FSB; for all traces, $M=64, \kappa=8,000$, and $N=8 . k=$ 1 for blue and unmarked trace and $k=25(\Delta f=96 \mathrm{MHz})$ for the green and red traces, which are cross-marked and circled-marked, respectively. 


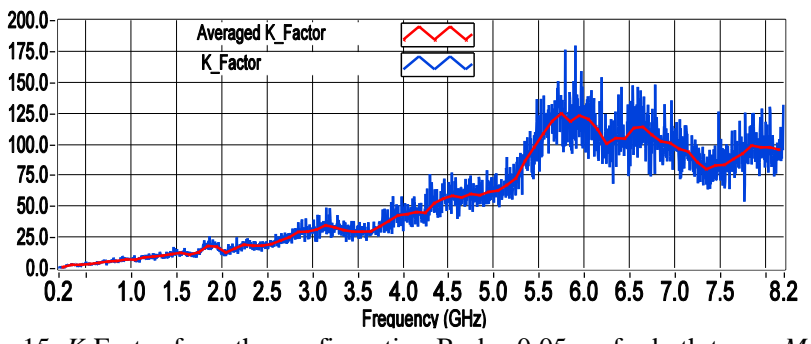

Fig. 15. $K$-Factor from the configuration $\mathrm{B}, \mathrm{d}=0.05 \mathrm{~m}$; for both traces $M=$ 64, $\kappa=2,000$, and $N=64 . k=1$ for blue and unmarked trace and $k=25(\Delta f$ $=96 \mathrm{MHz}$ ) for red and cross-marked trace.

Figs. (16)-(20) show results of measurements concerning the configuration $\mathrm{B}$ for $\mathrm{d}=0.3 \mathrm{~m}$.

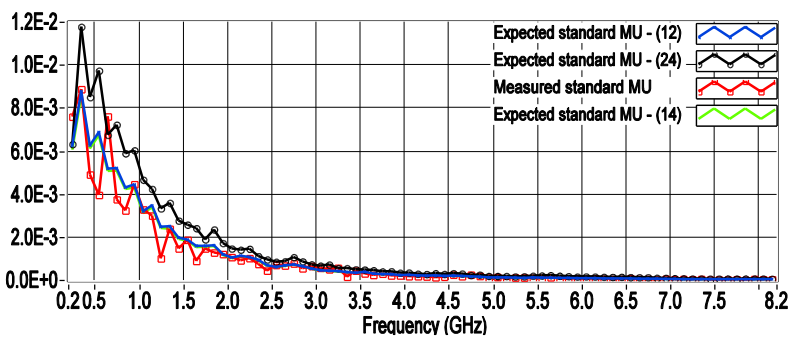

Fig. 16. Standard MU from the configuration $\mathrm{B}, \mathrm{d}=0.3 \mathrm{~m}$; for measured and expected standard uncertainties, $M=64, \kappa=2,000, N=8, n=8$, and $k$ $=25(\Delta f=96 \mathrm{MHz})$.

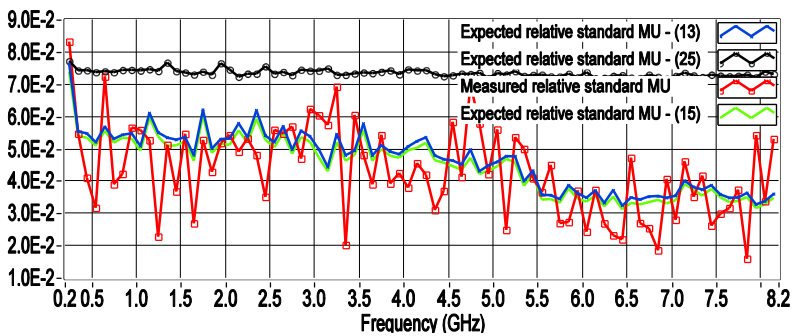

Fig. 17. Relative standard $\mathrm{MU}$ from the configuration $\mathrm{B}, \mathrm{d}=0.3 \mathrm{~m}$; for measured and expected standard uncertainties, $M=64, \kappa=2,000, N=8, n$ $=8$, and $k=25(\Delta f=96 \mathrm{MHz})$

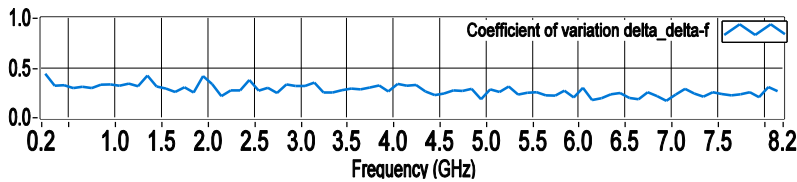

Fig. 18. $\mathrm{CV} \delta_{\Delta f}$ from the configuration $\mathrm{B}, \mathrm{d}=0.3 \mathrm{~m} ; N=8, k=25$ (96 $\mathrm{MHz})$.

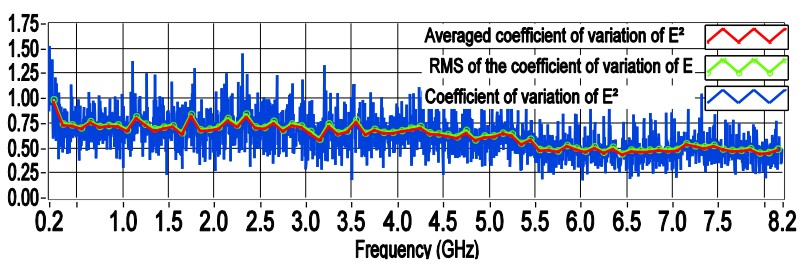

Fig. 19. CV $\delta_{E^{2}, f}$ from the configuration $\mathrm{B}(\mathrm{d}=0.03 \mathrm{~m})$, its average value, and RMS value in the FSB; for all traces, $M=64, \kappa=8,000$, and $N=8 . k=$ 1 for blue and unmarked trace and $k=25(\Delta f=96 \mathrm{MHz})$ for the green and red traces, which are cross-marked and circled-marked, respectively.

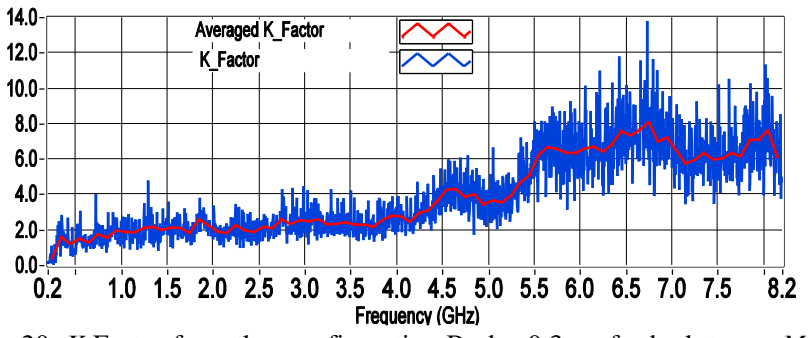

Fig. 20. $K$-Factor from the configuration $\mathrm{B}, \mathrm{d}=0.3 \mathrm{~m}$; for both traces $M=$ $64, \kappa=2,000$, and $N=64 . k=1$ for blue and unmarked trace and $k=25(\Delta f$ $=96 \mathrm{MHz}$ ) for red and cross-marked trace.

It is important to note that results from (12)-(13) are essentially the same as those from (14)-(15) except for $N<8$ as Fig. 10 shows. However, we consider ultimately the enhanced model (12)-(13) even though we believe that the variation (14)-(15) can generally be used for $N \geq 8$. Finally, we highlight that results in [21, Figs. 22-25], where no decimation was applied, did not match well because samples were partially correlated. The effect of a residual correlation is also appreciable in [21, Fig. 9] for $f>5 \mathrm{GHz}$.

\section{DISCUSSION}

The standard MU of the IL of an RC, as well as the relative standard uncertainty, is estimated for type A evaluation uncertainty; they are compared to the corresponding measured uncertainties. The estimate of the MU is obtained so that the uncertainty component $\sigma_{2}$ due to the nonuniformity of the field in the RC is highlighted and separately obtained, except the multiplying factor $\left(1+\delta_{s p, p, 0}^{2}\right)$ present in $\sigma_{1}$. The non-uniformity is affected by the load in the RC and it increases as the load increases. Such a component of uncertainty is connected to location, orientation, and polarization of both the transmitting and the receiving antennas for a given RC. The model gives good results at low frequencies as well. The non-uniformity of the field in an RC, which is estimated by $\sigma_{2}$ cannot be neutralized by the increase of sample dimension $N \cdot k$, even though, a marginal reduction of such a component of MU could be achieved by a widening of the FSB [11]. This aspect is very important when $\sigma_{2}$ has to be reduced. This could be the case where the effect of a strong load on the uniformity has to be reduced or when a very low total uncertainty is necessary. In [13], the PDFs of the interest sample statistics are theoretically achieved; the theory is applied to $\chi^{2}$ parent distributions with two or six degrees of freedom according to the sample statistic to be processed. The RVs, which are represented by the same amount of samples $N \cdot k \cdot p$ from hybrid stirring, are all assumed to be identically distributed (ID), so that the theoretical PDF is achieved, as well as the concerning uncertainty. It is specified that $M$ in [13] corresponds to $k$ in [11] and here, when MS and FS, but no position stirring, is considered whereas $M$ corresponds to the product $k \cdot p$ in [11], and in this paper when MS, FS, and position stirring are considered. It is important to highlight that the standard MU obtained here and in [11] is equivalent to that obtained in [13] for the average power, when the dependence on the frequency and the non-uniformity of the field are negligible in the FSB. 
For such measurement conditions, the averages $W$ and $W_{m p}$ certainly exhibit PDFs that can be approximated by a Gauss normal sampling distribution, according to the total number of acquired samples $N \cdot k \cdot p$, and the confidence intervals can also be obtained. However, one can note that the $N \cdot k \cdot p \mathrm{RVs}$ are not strictly ID as the IL is subject to the non-uniformity of fields inside an RC. Such variations are affected by the load of the RC as mentioned above. In other words, the RVs have all the same PDF type, but they have not strictly the same mean and standard deviation; that is, they are not strictly statistically equivalent. At low frequencies, the distributions of the field and power deviate from the idealized theoretical ones; therefore, the theory applied to $\chi^{2}$ is an approximation at low frequency. From the experimental point of view, when all samples are mixed up together, the total uncertainty is obtained. However, the assumption of RVs ID simplifies the theoretical developments and is certainly acceptable for small FSB and little non-uniformity of the field in the RC. The theory can also be extended to cases where fields are partially incoherent, i.e., cases where $K>0$ [13, pag. 31]. In [13], many PDFs of practical interest and the related uncertainties are achieved, as well as the corresponding confidence intervals, including the PDF and the uncertainty of the maximum value for both field and power. The standard "uncertainty of the uncertainty" is also achieved. Results from some applications expected for the standard [1] are also shown. Finally, we believe that the averages $W$ and $W_{m p}$ exhibit PDFs approximately normal in all common usable measurement conditions in RCs including loaded RCs [20], [24]. Similarly, we believe that the assumption of RVs ID made in [13], along with the extension of the theory to cases where $K>0$, causes an acceptable approximation in all common usable measurement conditions in RCs including loaded RCs.

\section{CONCLUSIONS}

In this paper, an enhancement of the previous model for the standard $\mathrm{MU}$ in an $\mathrm{RC}$ is shown: it is de facto a generalization of the previous model. A majorant of the standard MS is derived as well. By results from measurements, it is shown that enhanced model works well for both high and low frequencies. It includes the previous model as a particular case and does not require specific conditions for its validity. The majorant is based on a weak condition on the $\mathrm{CV}$ of the parameter to be measured, i.e., it has to be less than or equal to one. The majorant, which just corresponds to the previous model, could be used when the abovementioned $\mathrm{CV}$ is less than one and a conservative margin is considered for the statistical fluctuation. However, it does not work well at low frequencies, where the condition for its validity does not hold true. Finally, the comparison between the model shown here and that in [13] was discussed; it is concluded that both approaches are practically sound.

\section{REFERENCES}

[1] IEC 61000-4-21, Electromagn. Compat. (EMC), Part 4-21: Testing and measurement techniques - Reverberation chamber test methods,
International Electrotechnical Commission, Geneva, Switzerland, 2011.

[2] U. Carlberg, P. S. Kildal, and J. Carlsson, "Numerical study of position stirring and frequency stirring in a loaded reverberation chamber," IEEE Trans. Electromagn. Compat., vol. 51, pp. 12-17, 2009.

[3] D.A. Hill, Electromagnetic Fields in Cavities: Deterministic and Statistical Theories. New York: IEEE Press, 2009.

[4] X. Chen "Measurement uncertainty of antenna efficiency in a Reverberation Chamber," IEEE Trans. Electromagn. Compat., vol. 55, pp. 1331-1334, Dec. 2013.

[5] I.D. Flintoft, G.C. R. Melia, M.P. Robinson, J.F. Dawson, and A.C. Marvin, "Rapid and accurate broadband absorption cross-section measurement of human bodies in a reverberation chamber", IOP Measurement Science and Technology, vol. 26, no. 6, art. no. 065701, pp. 1-9, May 2015.

[6] A. Gifuni, G. Ferrara, A. Sorrentino, and M. Migliaccio, "Analysis of the measurement uncertainty of the absorption cross section in a reverberation chamber", IEEE Trans. Electromagn. Compat., vol. 57, no. 5, pp. 1262-1265, Oct. 2015.

[7] A. Gifuni, I.D. Flintoft, Simon J. Bale, G.C. R. Melia, and A.C. Marvin, "A theory of alternative methods for measurements of absorption cross section and antenna radiation efficiency using nested and contiguous reverberation chambers", IEEE Trans. Electromagn. Compat., vol. 58, pp. 678-685, June 2016.

[8] C.L. Holloway, H.A. Haider, R.J. Pirkl, W.F. Yong, D.A. Hill, J. Ladbury, "Reverberation chamber techniques for determining the radiation and total efficiency of antennas," IEEE Trans. Electromagn. Compat., vol.60, pp.1758-1770, April 2012.

[9] A. Gifuni and S. Perna, "Analysis on the calculation of the inverse discrete Fourier transform (IDFT) of passband frequency response measurements in terms of lowpass equivalent response," Prog. In Electromagn. Research, vol. 160, 63-69, 2017.

[10] X. Zhang, M. Robinson, I.D. Flintoft, and J.F. Dawson "Inverse Fourier transform technique of measuring averaged absorption cross section in the reverberation chamber and Monte Carlo study of its uncertainty," Electrom. Compat., IEEE Intern. Symp. EMC Europe, pp. 263-267, 2016, DOI:10.1109/EMCEurope.2016.7739161.

[11] A. Gifuni, L. Bastianelli, F. Moglie, V. M. Primiani, and G. Gradoni, "Base-case model for measurement uncertainty in a reverberation chamber including frequency stirring," IEEE Trans. Electromagn. Compat., DOI: 10.1109/TEMC.2017.2763627.

[12] B. N. Taylor and C. E. Kuyatt, "Guidelines for Evaluating and Expressing the Uncertainty of NIST Measurement Results," NIST Tech. Note 1297, Sept. 1994.

[13] L. R. Arnaut, Measurement Uncertainty in Reverberation Chambers I. Sample Statistics, $2_{\text {nd. }}$ ed., NPL Report TQE2, pp. 1-136, Dec. 2008.

[14] C. Lemoine, E. Amador, and P. Besnier, "On the $K$-factor estimation for Rician channel simulated in reverberation chamber," IEEE Trans. Antennas Propag., vol. 59, no. 3, pp. 1003-1012, Mar. 2011.

[15] C.L. Holloway, D.A. Hill, J.M. Ladbury, and G. Kapke, "Requirements for an effective reverberation chamber: unloaded or loaded," IEEE Trans. Electromagn. Compat., vol.48, pp.187-194, Feb. 2006.

[16] K.A. Remley, R.J. Pirkl, C.M. Wang, D. Senic, A.C. Homer, M.V. North, M.G. Becker, R.D. Horansky, and C.L. Holloway, "Estimating and correcting the device-under-test transfer function in loaded reverberation chambers for over-the-air-tests," IEEE Trans. Electromagn. Compat., vol. 59, pp. 1724-1734, Dec. 2017.

[17] V. Rajamani, C.F. Bunting, and J.C. West "Stirred-Mode operation of reverberation chambers for EMC testing," IEEE Trans. Electromagn. Compat., vol.61, pp.2759-2764, Oct. 2012.

[18] L.R. Arnaut, "Effect of local stir and spatial averaging on measurement and testing in mode-tuned and mode-stirred reverberation chambers," IEEE Trans. Electromagn. Compat., vol. 43, pp. 305-325, August 2001.

[19] D. Blumenfeld, Operations Research Calculations Handbook, CRC Press, New York, 2001.

[20] A. Papoulis, "Probability, random variables and Stochastic Process," New York: McGraw-Hill, 1991. 
[21] A. Gifuni, L. Bastianelli, G. Gradoni, M. Migliaccio, F. Moglie, and V. Mariani Primiani, "Applicability of measurement uncertainty models in a reverberation chamber including frequency stirring," in Proc. IEEE Int. Symp. Electromagn. Compat. Sig. Pow. Integr. (EMC-SIPI), Long Beach, CA, USA, 2018.

[22] K.A. Remley, J. Dortmans, C. Weldon, R.D. Horansky, T.B. Meurs, C.-M. Wang, D.F. Williams, and C.L. Holloway, "Configuring and verifying reverberation chambers for testing cellular wireless devices," IEEE Trans. Electromagn. Compat., vol. 58, no. 3, pp. 661-671, June, 2016.

[23] L.R. Arnaut, M.I. Andries, J. Soil, P. Besnier "Evaluation method for the probability distribution of the quality factor of mode-stirred reverberation chambers," IEEE Trans. Electromagn. Compat., vol. 43, pp. 305-325, August 2001.

[24] A. Gifuni, A. Sorrentino, G. Ferrara, M. Migliaccio, "An estimate of the probability density function of the sum of a random number $N$ of independent random variables," Journ. of Comput. Engineering, Hindawi Publishing Corporation, vol. 2015, 12 pages, 2015. doi:10.1155/2015/801652. 